\title{
Correction: Endostatin and ST2 are predictors of pulmonary hypertension disease course in infants
}

\author{
Megan Griffiths $(\mathbb{D} \cdot$ Jun Yang · Allen D. Everett · Jacky M. Jennings · Grace Freire • Monica Williams · Melanie Nies • \\ Sharon A. McGrath-Morrow · Joseph M. Collaco
}

Published online: 2 June 2020

(c) The Author(s), under exclusive licence to Springer Nature America, Inc. 2020

Correction to: Journal of Perinatology

https://doi.org/10.1038/s41372-020-0671-8

published online 4 May 2020

Since online publication of this article, the authors noticed that there was an error in the funding information. The correct text is provided below. The authors apologize for this error.

Funding MG was supported by the Pediatric Scientist Development Program. The Pediatric Scientist Development Program is supported by Award Number K12-HD000850 from the Eunice Kennedy Shriver
National Institute of Child Health and Human Development. JMC received support from a Johns Hopkins All Children's Hospital Foundation Institutional Research Grant. The work was supported in part by a Johns Hopkins All Children's Foundation Institutional Research Grant (PI: N.A. Goldenberg; Johns Hopkins "iPICS" prospective multi-cohort and biobanking study of pediatric acute and chronic health conditions).

Author contributions MG, JMC, and ADE planned the project, analyzed the data, and wrote the manuscript; MG and JY performed the experiments and interpreted the results; MG and JMC performed statistical analysis; JY, ADE, MN, MW, JMJ, GF, SMM, and JMC recruited participants and performed research; and all authors reviewed, revised, and approved the manuscript for submission. 